\title{
Other T- and B-Aggressive Lymphomas and Lymphomas Associated with HIV
}

\author{
Kai Hübel and Silvia Montoto
}

\subsection{Burkitt Lymphoma (BL)}

\subsubsection{Definition and Epidemiology}

BL accounts for around $2 \%$ of all adult NHL with a higher incidence in patients with immunodeficiency and in patients who are HIV positive. There is an endemic pediatric subtype in Equatorial Africa which is strongly associated with EBV. The clinical course of BL usually is highly aggressive with a Ki67 expression of nearly $100 \%$ requiring prompt institution of therapy.

\subsubsection{Diagnosis}

A tissue biopsy/cytology sample is mandatory for the diagnosis. The hallmark of the tumor is the rearrangement of a gene encoding MYC, but the diagnosis requires a combination of morphology, immunophenotype, and genetic analysis.

\section{K. Hübel $(\bowtie)$}

Department I of Internal Medicine, University of

Cologne, Cologne, Germany

e-mail: kai.huebel@uni-koeln.de

\section{S. Montoto}

Department of Haemato-Oncology, St.

Bartholomew's and The Royal London NHS Trust, Queen Mary University of London, London, UK

\subsubsection{Risk Factors}

Several studies have identified risk factors for poor outcome. Beside older age, advanced stage, and comorbidities, such risk factors are an elevated serum $\mathrm{LDH}$, failure to achieve $\mathrm{CR}$, anemia, CNS involvement, and BM infiltration.

\subsubsection{First-Line Treatment}

The optimal first-line therapy in BL has not been defined yet. To achieve a fast and stable remission, an intensive regimen combining several compounds is used in most centers. Combinations of RTX, doxorubicin (DOX), alkylators, VCR, and VP with direct therapy to prevent CNS disease are highly active.

The HOVON CODOX-M/IVAC regimen (CY, DOX, VCR, MTX, IFO, Ara-C, and VP) achieved a 2-year EFS of $65 \%$ and a 2 -year OS of $73 \%$ and is very active especially in high-risk patients (Mead et al. 2002).

The B-ALL protocol (MTX, Ara-C, CY, VP, IFO) of the German GMALL study group achieved a CR in $88 \%$ of patients, with a 5-year PFS of $71 \%$ and a 5-year OS of $80 \%$ (Hoelzer et al. 2014). This means that a significant portion of patients have a chance to get cured with firstline therapy.

Recently, using modifications of R-EPOCH (RTX, VP, VCR, CY, DOX), high activity in BL was reported (Roschewski et al. 2017). 


\subsubsection{Autologous HSCT}

There are several studies exploring the role of autoHSCT in first remission. In a prospective trial, the HOVON group treated 27 patients with 2 cycles of intensive induction followed by BEAM-conditioned auto-HSCT for those patients achieving at least a PR (van Imhoff et al. 2005). The 5-year EFS and OS was $73 \%$ and $81 \%$, respectively. In a retrospective analysis of 117 patients receiving auto-HSCT as part of first-line therapy between 1984 and 1994, patients in CR at time of transplant had a 3-year OS of $72 \%$ (Sweetenham et al. 1996). In the relapse situation, patients who were chemotherapy-sensitive had a 3-year OS of $37 \%$ following auto-HSCT compared to just $7 \%$ for those who were chemotherapy resistant (Sweetenham et al. 1996). In summary, auto-HSCT in BL is feasible, but there is no documented advantage compared to standard combination chemotherapy for patients responding sufficiently to first-line treatment. Auto-HSCT may be used to optimize remission in patients with insufficient response or as bridging to allo-HSCT. In the relapse setting, given the intensive regimens usually used as first-line treatment, the difficulty lies in achieving a response good enough to proceed to auto-HSCT and to collect HSC; hence, auto-HSCT is rarely used in $\mathrm{BL}$.

\subsubsection{Allogeneic HSCT}

(Peniket et al. 2003)

\begin{tabular}{|l|l|}
\hline Indicated in & CR $\geq 2$ \\
\hline Donor & MRS $>$ MUD > MMUD \\
\hline Conditioning & RIC > MAC \\
\hline TRM (1 year) & $30 \%$ \\
\hline OS (5 year) & $30-40 \%$ \\
\hline DFS & $35 \%$
\end{tabular}

\subsection{Lymphoblastic Lymphoma (LBL)}

\subsubsection{Definition and Epidemiology}

LBL is an aggressive neoplasm of precursor B cells (B-LBL) or T cells (T-LBL) with features of acute leukemia. It accounts for approximately $2 \%$ of all NHL. In adults, around $90 \%$ of all LBL are T-LBL.

\subsubsection{Diagnosis}

The diagnosis is based on a LN biopsy. T-LBL is usually TdT-positive with a variable expression of other T-cell markers (CD7 and CD3 are often positive).

\subsubsection{Risk Factors}

At this time, no convincing prognostic model for these patients is available. Several studies tried to identify risk factors; the following may be associated with an unfavorable outcome: elevated $\mathrm{LDH}, \mathrm{BM}$ or CNS involvement, and stage IV disease. The role of MRD in LBL has not been defined yet, but as we learned from acute leukemias and other lymphomas, persisted MRD positivity might be a predictor of poor outcome.

\subsubsection{First-Line Treatment}

Standard approaches for patients with LBL are adapted to ALL protocols. These regimens contain multiple drugs, such as CY, MTX, VCR, Ara-C, thioguanine, L-asp, VP, nitrosoureas, and anthracyclines. With these protocols, a CR rate of $80 \%$ and a DFS of $56 \%$ have been reported (Estey et al. 2008).

\subsubsection{Autologous HSCT}

There are only very few studies evaluating the role of auto-HSCT in LBL. In CR1, the use of autoHSCT as a consolidation may improve relapsefree survival but has no effect on OS (Sweetenham et al. 2001). In another study in 128 patients with LBL receiving auto-HSCT, RR at 5 years was $56 \%$ (Levine et al. 2003). No documented role in more advanced disease $>\mathrm{CR} 1$ is reported either. In 
conclusion, data for auto-HSCT in LBL are too scarce to come to firm conclusions.

\subsubsection{Allogeneic HSCT}

There is also no established role for allo-HSCT in patients with LBL. Compared to auto-HSCT, allo- HSCT is associated with a higher TRM but lower RR. In 76 patients receiving allo-HSCT, 5-year RR was 34\% (Levine et al. 2003). In this retrospective study, there was no significant difference in OS at 1 year and 5 years between autoHSCT and allo-HSCT. In general, the indication for allo-HSCT should be based on risk factors, remission, and MRD.

\subsection{Peripheral T-Cell Lymphomas (PTCLs)}

\subsubsection{Definition and Epidemiology}

PTCLs are a very heterogeneous group of lymphomas originating from the T-cell lineage. They account for approximately $10-15 \%$ of all NHL. Because of this low incidence, large randomized studies are difficult to perform.

\subsubsection{Diagnosis}

The diagnosis, as in any NHL, should be based on a LN biopsy. The differential diagnosis between PTCL and other types of T-NHL is crucial for the outcomes, and in some specific cases, the treatments are very different.

\subsubsection{Risk Factors}

The IPI is the most commonly used prognostic tool in PTCL. The following factors are associated with worse outcome: age $>60$ years, ECOG $>1$, elevated LDH, stages II-IV, and extranodal involvement $>1$ site. In anaplastic large cell lymphoma (ALCL), the tumors are categorized in ALK+ or ALK- with better prognosis for ALK+ lymphomas.

\subsubsection{First-Line Treatment}

The primary goal of first-line treatment is to get a deep and continuing remission. Standard regimens are anthracycline-containing combinations like CHOP or CHOEP, achieving a 3-year EFS of $50-70 \%$ and a 3-year OS of 75-80\% (Schmitz et al. 2010). In the relapse situation, the overall prognosis of PTLC is dismal, and the optimal treatment for these patients has not been defined yet. Relapse patients not able to receive intensive treatment including HSCT may be offered singleagent therapy, e.g., gemcitabine, or in case of CD30 expression, brentuximab vedotin.

\subsubsection{Autologous HSCT (Kyriakou} et al. 2008; d'Amore et al. 2012; Kewalramani et al. 2006; Wilhelm et al. 2016)

\begin{tabular}{|l|l|}
\hline Indicated in & CR1 (IPI > 1), CR $\geq 2$ \\
\hline Conditioning & BEAC, BEAM, LEAM, CBV \\
\hline TRM (1 year) & $5-7 \%$ \\
\hline REL (3 years) & $40-50 \%$ \\
\hline OS (5 years) & $70 \%(C R 1), 50 \%(C R \geq 2)$ \\
\hline PFS (5 years) & $50 \%(C R 1), 20-35 \%(C R \geq 2)$
\end{tabular}

87.3.6 Allogeneic HSCT (Schmitz et al. 2014, 2018; Dodero et al. 2012)

\begin{tabular}{|l|l|}
\hline Indicated in & CR $\geq 2$, relapse post auto-HSCT \\
\hline Donor & MRS $>$ MUD $>$ MMUD \\
\hline Conditioning & RIC $>$ MAC \\
\hline GVHD prophylaxis & CSA, CSA-MTX, CSA-MMF \\
\hline Graft failure & $<10 \%$ \\
\hline TRM (1 year) & $20-25 \%$ \\
REL (3 years) & $20-40 \%$ \\
\hline OS (3 years) & $40-60 \%$ \\
\hline PFS (3 years) & $30-50 \%$
\end{tabular}

\subsection{HIV-Associated Lymphomas}

\subsubsection{Definition and Epidemiology}

Patients infected with HIV have an increased risk of developing NHLs as compared to the general population. The most frequent subtypes are 
DLBCL and BL. Both are AIDS-defining illnesses, while HL is one of the non-AIDS-defining malignancies.

\subsubsection{Risk Factors}

In the era of combined antiretroviral therapy (cART), the risk factors that determine prognosis in patients with HIV-related lymphoma are the same as in the general population and, hence, depend on the subtype of lymphoma, rather than on HIV-related factors.

\subsubsection{First-Line Therapy}

The optimal therapy for these patients has not been defined yet, but since the availability of cART, the outcome of HIV-lymphoma has considerably improved. The consequent use of cART during therapy is of major importance for successful treatment. In first-line treatment, patients will receive treatment comparable with HIVnegative patients. The indication for HSCT has to be discussed in the relapse situation.

\subsubsection{Autologous HSCT (Hübel et al. 2017; Diez-Martin et al. 2009; Balsalobre et al. 2009)}

\begin{tabular}{l|l} 
Indicated in & $\begin{array}{l}\text { CR } \geq 2 \text {; same indications as in the } \\
\text { general population with the same type } \\
\text { of lymphoma }\end{array}$ \\
\hline $\begin{array}{l}\text { Conditioning } \\
\text { BRAC, BEAM, LEAM, CBV } \\
\text { (1 year) }\end{array}$ & $5-7 \%$ \\
\hline $\begin{array}{l}\text { REL } \\
\text { (3 years) }\end{array}$ & $30-40 \%$ \\
\hline $\begin{array}{l}\text { OS (5 years) } \\
\text { PFS (5 years) }\end{array}$ & $50-60 \%$ \\
\hline PFS $60 \%$
\end{tabular}

\subsubsection{Allogeneic HSCT}

Experience on the use of allo-HSCT in patients with lymphoma and HIV infection is very limited, and no definitive recommendation can be given at this time. There are some case reports or small retrospective analysis showing that alloHSCT in HIV-positive patients using MRD, MUD, or CB is feasible, but application of cART and viroimmunological reconstitution is a matter of debate. In a recent report of five HIV-positive patients who underwent allo-HSCT with various hematologic malignancies, there was no TRM or major infections (Mulanovich et al. 2016). HIV virus load remained undetectable with continuous cART. Three patients relapsed 6,7 , and 13 months after transplant, and two were alive and well after 42 and 55 months.

\section{References}

Balsalobre P, Diez-Martin JL, Re A, et al. Autologous stem-cell transplantation in patients with HIV-related lymphoma. J Clin Oncol. 2009;27:2192-8.

d'Amore F, Relander T, Lauritzsen GF, et al. Up-front autologous stem-cell transplantation in peripheral T-cell lymphoma: NLG-T-01. J Clin Oncol. 2012;30:3093-9.

Diez-Martin JL, Balsalobre P, Re A, et al. Comparable survival between HIV+ and HIV- non-Hodgkin and Hodgkin lymphoma patients undergoing autologous peripheral blood stem cell transplantation. Blood. 2009;113:6011-4.

Dodero A, Spina F, Narni F, et al. Allogeneic transplantation following a reduced-intensity conditioning regimen in relapsed/refractory peripheral T-cell lymphomas: long-term remissions and response to donor lymphocyte infusions support the role of a graftversus-lymphoma effect. Leukemia. 2012;26:520-6.

Estey EH, Faderl SH, Kantarjian H. Acute leukemias. Berlin: Springer; 2008. p. 167-76.

Hoelzer D, Walewski J, Dohner H, et al. Improved outcome of adult Burkitt lymphoma/leukemia with rituximab and chemotherapy: report of a large prospective multicenter trial. Blood. 2014;124:3870-9.

Hübel K, Re A, Boumendil A, Finel H, Hentrich M. HIVassociated lymphoma and autologous stem cell transplantation in the rituximab era: a retrospective analysis on indication, outcome and risk factors by the EBMT Lymphoma Working Party. Bone Marrow Transplant. 2017;52(S1):141.

Kewalramani T, Zelenetz AD, Teruya-Feldstein J, et al. Autologous transplantation for relapsed or primary refractory peripheral T-cell lymphoma. Br J Haematol. 2006; 134:202-7.

Kyriakou C, Canals C, Goldstone A, et al. High-dose therapy and autologous stem-cell transplantation in angioimmunoblastic lymphoma: complete remission at transplantation is the major determinant of 
Outcome-Lymphoma Working Party of the European Group for Blood and Marrow Transplantation. J Clin Oncol. 2008;26:218-24.

Levine JE, Harris RE, Loberiza FR Jr, et al. A comparison of allogeneic and autologous bone marrow transplantation for lymphoblastic lymphoma. Blood. 2003;101:2476-82.

Mead GM, Sydes MR, Walewski J, et al. An international evaluation of CODOX-M and CODOX-M alternating with IVAC in adult Burkitt's lymphoma: results of United Kingdom Lymphoma Group LY06 study. Ann Oncol. 2002;13:1264-74.

Mulanovich VE, Desai PA, Popat UR. Allogeneic stem cell transplantation for HIV-positive patients with hematologic malignancies. AIDS. 2016;30:2653-7.

Peniket AJ, Ruiz de Elvira MC, et al. An EBMT registry matched study of allogeneic stem cell transplants for lymphoma: allogeneic transplantation is associated with a lower relapse rate but a higher procedurerelated mortality rate than autologous transplantation. Bone Marrow Transplant. 2003;31:667-78.

Roschewski M, Dunleavy K, Abramson JS, Link BK. Risk-adapted therapy in adults with Burkitt lymphoma: results of NCI 9177, a multicenter prospective phase II study of DA-EPOCH-R. Blood. 2017;130(Suppl 1):188.

Schmitz N, Trumper L, Ziepert M, et al. Treatment and prognosis of mature T-cell and NK-cell lymphoma: an analysis of patients with T-cell lymphoma treated in studies of the German High-Grade Non-Hodgkin Lymphoma Study Group. Blood. 2010;116:3418-25.
Schmitz N, Wu HS, Glass B. Allogeneic transplantation in T-cell lymphomas. Semin Hematol. 2014;51:67-72.

Schmitz N, Lenz G, Stelljes M. Allogeneic hematopoietic stem cell transplantation (HSCT) for T-cell lymphomas. Blood. 2018. https://doi.org/10.1182/ blood-2018-01-791335. [Epub ahead of print].

Sweetenham JW, Pearce R, Taghipour G, et al. Adult Burkitt's and Burkitt-like non-Hodgkin's lymphoma-outcome for patients treated with high-dose therapy and autologous stem-cell transplantation in first remission or at relapse: results from the European Group for Blood and Marrow Transplantation. J Clin Oncol. 1996;14:2465-72.

Sweetenham JW, Santini G, Qian W, et al. High-dose therapy and autologous stem-cell transplantation versus conventional-dose consolidation/maintenance therapy as postremission therapy for adult patients with lymphoblastic lymphoma: results of a randomized trial of the European Group for Blood and Marrow Transplantation and the United Kingdom Lymphoma Group. J Clin Oncol. 2001;19:2927-36.

van Imhoff GW, van der Holt B, MacKenzie MA, et al. Short intensive sequential therapy followed by autologous stem cell transplantation in adult Burkitt, Burkitt-like and lymphoblastic lymphoma. Leukemia. 2005;19:945-52.

Wilhelm M, Smetak M, Reimer P, et al. First-line therapy of peripheral T-cell lymphoma: extension and longterm follow-up of a study investigating the role of autologous stem cell transplantation. Blood Cancer J. 2016;6:e452.

Open Access This chapter is licensed under the terms of the Creative Commons Attribution 4.0 International License (http://creativecommons.org/licenses/by/4.0/), which permits use, sharing, adaptation, distribution and reproduction in any medium or format, as long as you give appropriate credit to the original author(s) and the source, provide a link to the Creative Commons license and indicate if changes were made.

The images or other third party material in this chapter are included in the chapter's Creative Commons license, unless indicated otherwise in a credit line to the material. If material is not included in the chapter's Creative Commons license and your intended use is not permitted by statutory regulation or exceeds the permitted use, you will need to obtain permission directly from the copyright holder. 